\title{
Quality of life at work (QWL) and performance: towards new forms of well-being at work
}

\section{Prof Bouchra BELMOUFFEQ}

Laboratoire de Recherche Nouvelle Economie et Développement (LARNED)

\begin{abstract}
The technical and socio-organizational changes that deeply mark the economic world the expectations of employees in terms of availability, mobility and flexibility are growing and invite us to rethink the modes of governance of organizations. Robotization and digitization certainly make work abstract and more creative, but metamorphosis means crossing profitability performance with productivity of employees and will-being at work.

New technologies reducing the border between family and work space, more than half of executives work outside of the company, as it is difficult to escape the pressure of ", all the time, right now". Dead work tends to replace living work and challenges the human resources function which is required to accompany transformations in order to preserve a favorable, motivating and rewarding social climate in order to acquire highly qualified human potential and ensure its conservation and development.

The HR manager is not a psychologist or a psychotherapist. Its mission is not to resolve the personal, relational or emotional difficulties of its employees. However, its responsibility is to generate the best working conditions to boost their performance. He is called upon to create a pleasant, more agile and more flexible environment where everyone will give the best of himself.
\end{abstract}

Keywords-economic world, performance with productivity, psychotherapist.

Qualité de vie au travail (QVT) et performance : vers des nouvelles formes de bien être au travail Prof Bouchra BELMOUFFEQ

Résumé- Les mutations techniques et socio-organisationnelles qui marquent profondément l'actualité du monde économique et les attentes croissantes des collaborateurs en termes de disponibilité, de mobilité et de flexibilité invitent à repenser les modes de gouvernance des organisations. Robotisation, numérisation rendent certes le travail abstrait et créatif mais la métamorphose impose de croiser les calculs de la rentabilité aux formes appropriés capables d'aligner entre le bien être au travail et la productivité des collaborateurs.

Les nouvelles technologies réduisant la frontière entre la famille et le l'espace de travail, plus de la moitié des cadres travaillent en dehors des murs de l'entreprise, tant il est difficile d'échapper à la pression du ", tout le temps, tout de suite". Le travail mort tend à remplacer le travail vivant et interpelle la fonction ressources humaine qui est tenu accompagner les transformations afin de préserver un climat social favorable, motivant et valorisant pour se doter d'un potentiel humain hautement qualifié et assurer sa conservation et son développement.

Le manager RH n'est pas un psychologue ou un psychothérapeute. Sa mission ne consiste pas à régler les difficultés personnelles, relationnelles ou émotionnelles de ses collaborateurs. Toutefois sa responsabilité consiste à générer les meilleures conditions de travail pour booster leur rendement. Il est appelé à créer un cadre agréable, plus agile et plus flexible où chacun donnera le meilleur de lui-même. 


\section{INTRODUCTION}

Pendant longtemps, le travail a été considéré par les salariés comme un simple gagne-pain, un moyen de subvenir à ses besoins sans aucune considération aux conditions de son exercice. Aujourd'hui le travail est considéré comme vecteur d'épanouissement et de réalisation de soi : les salariés cherchent à s'y sentir bien.

L'entreprises, agent économique rationnel privilégie les modèles ou l'output performance est garanti, un modèle selon lequel la recherche de la performance prime dans toute les décisions (ENRIQUEZ (1997, pp. 118-119), l'investissement dans le bien-être des employés, et plus globalement la prise en compte de celui-ci dans les décisions organisationnelles, ne se justifie que si la qualité de vie au travail est susceptible d'impacter la performance.

La qualité de travail est définie comme un indice du caractère et de la situation de travail. Les aspects en relation avec le bien-être au travail constituent notamment des composantes importantes de la qualité du travail ${ }^{1}$. Page et Vella-Brodrick 2019, p. 450) ont recommandé de recourir à une conception plus large du bien-être qui intègrerait la mesure du fonctionnement psychologique des salariés. Le bien-être est un état de différents facteurs séparées par exemple : la santé, la réalisation de soi...

Pour simplifier l'analyse nous résumons les facteurs de bien être en deux parties essentielles : le facteur physique (la sensation d'une bonne santé) et le facteur psychologique (sensation de satisfaction des conditions matérielles, sociales, professionnelles....)

Nous cherchons dans ce papier à expliquer la performance (variable expliquée) par l'investissement en facteur physique nommé ci-après $(\mathrm{X}=$ variable explicative 1$)$ et le facteur psychologique désigné $(\mathrm{Y}=$ variable explicative 2$)$ pour justifier le niveau (P) de la performance souhaité.

Donc, Si l'entreprise investit en bien être au travail cela permet il d'augmenter la performance de ses salariés ? Le sens de variation de la performance $(\mathrm{P})$ suit il le facteur physique $(\mathrm{X})$ ou le facteur psychologique? ou les deux à la fois ? et à quel dosage (degré) ?

Pour vérifier le lien de causalité entre ces variables nous menons une enquête auprès des cadres-salariés. Nous allons emprunter la démarche hypothético-déductive qui consiste à formuler des hypothèsess afin d'en déduire des conséquences observables futures (démarche prédictive), et passées (démarche rétro- diction ), permettant d'en déterminer la validité selon le modèle conceptuel suivant :

Le modèle conceptuel de notre recherche est défini par le régression linéaire suivante :

$\mathbf{P}=\mathbf{a}(\mathbf{X})+\mathbf{b}(\mathbf{Y})+\mathfrak{£}$

Où $\int \mathrm{P}=$ niveau de la performance prévu ou souhaité,

$\{\mathrm{X}=$ facteur physique (Coefficient de signification 1)

Y Facteur psychologique (Coefficient de signification 2)

\section{\% Bien-être physique}

Acceptation de soi

Relations positives avec autrui

Autonomie

Maitrise de l'environnement

Sens de la vie

Motivation

Communication interne

Satisfaction

\section{$\%$ Bien-être physique}

Acceptation de soi

Relations positives avec autrui

Autonomie

Maitrise de l'environnement

Sens de la vie

Motivation

Communication interne

Satisfaction
Bonne Qualité de

vie au travail<smiles>C1CC2CCC1C2</smiles>

Performance

Dans la tâche<smiles>C1CCCC1</smiles>

Performance

Économique

Notre recherche se subdivise en deux sections :

${ }^{1}$ Chambre des salariés de Luxemburg, Une analyse des données du Quality of work Index Luxembourg Newsletter $\mathrm{N}^{\circ}$

2/2015, 16 mars 2015 
- La première section sera consacrée à une revue de littérature, nous commençons par exposer certaines notions théoriques relatives au concept de bien-être et de bien-être au travail avant de procéder à une revue des études portant sur la relation entre le bien-être et la performance.

- La seconde section sera empirique essayons de répondre aux questions de recherche en s'appuyant sur le modèle susmentionné. Nous analysons ensuite les données collectées grâce à un questionnaire.

Nous bouclons ce travail par une discussion et une synthèse des résultats obtenus.

Section I : Le lien entre bien-être au travail et la performance économique

Le bien être au travail est définit comme l'ensemble des facteurs et /ou les conditions dans lesquelles un travail est exercé. La législation du travail impose aux employeurs d'assurer les conditions d'hygiène, de santé/sécurité, et de combattre les risques professionnels auxquels les salariés peuvent être exposés. Par ailleurs la charge psychosociale occasionnée par le rythme du travail (stress), l'ergonomie, l'embellissement des lieux de travail révèlent moins d'importance.

\section{I-Cadre conceptuel et théorique d'analyse}

\section{I.1-Soubassements théoriques}

De nombreuses théories ont démontré l'importance du capital humain dans l'entreprise, et ont souligné que des salariés satisfaits de leur travail et environnement de travail sont également des salariés plus performants. En raison de l'abondance des courants de pensée théorique, nous allons nous limiter aux principales approches ayant abordé le lien entre les conditions et la productivité du travail dans l'ordre chronologique.

Avant la crise de surproduction (1929), la demande dépassait l'offre et les entreprises cherchaient à optimiser leur système de production pour satisfaire la demande croissante. C'est dans ce sens que l'hypothèse taylorienne qui ne prenait en compte que les techniques et les conditions matérielles du travail pour améliorer la productivité, au prix de la deshumanisation des travailleurs, trouvait sa justification.

Cette approche réductionniste de la performance à sa posture économique a été complétée par Elton MAYO, en étudiant notamment le comportement des salariés au travail. Ses principales contributions sont le fruit de recherches empiriques menées au sein de la Western Electric. Mayo complète l'hypothèse taylorienne selon laquelle les conditions matérielles, techniques, du travail influencent la productivité en y associant des avantages pour les employés; salaires acceptables, environnement agréable, horaires bien étudiés, sécurité au poste, sécurité de l'emploi...

Les enquêtes à la Western Electric sont célèbres non seulement parce qu'elles ont introduit une rupture avec le mode de raisonnement taylorien mais surtout parce qu'elles ont ouvert la voie

à un renouvellement de la pensée sur les comportements humains au sein des organisations.

Les conclusions de Mayo soulignent l'existence d'un lien étroit entre sentiments

et comportements, de même que l'importance du poids du groupe sur les comportements individuels ou l'impact des normes collectives sur le rendement des ouvriers et donc sur la performance de l'organisation.

Quand à MASLOW, le rendement des salariés varie en fonction du degré de satisfaction de leurs besoins hiérarchisés. Ce cheminement explique les attentes des salariés tout au long de leurs carrières. Au début ils éprouvent le besoin d'un salaire pour vivre et un emploi avec une certaine sécurité (type de contrat, déclarations, Assurance-maladies...etc). Ensuite ils cherchent d'appartenir à un groupe, puis ils cherchent l'estime de leurs pairs, de leur chef. Après des années d'expérience, l'important est de s'accomplir véritablement. En cas d'accident professionnel, la perte d'un emploi, les besoins physiologiques et de sécurité reviennent en premier plan : avoir un travail et un salaire pour vivre ${ }^{2}$.

Pour booster la performance, il est opportun de comprendre les besoins de ces salariés et d'y apporter les réponses : Recherchent-ils la sécurité d'emploi (besoin de sécurité) ? Ont-ils besoin d'un esprit d'équipe plus présent (estime) -

2 Yves DURAN\& Zwi SEGAL . La motivation, une compétence qui se développe: Guide pour développer la motivation et l'engagement au travail Edition Pearson, Décembre 2015 
Souhaitent-ils atteindre des seuils objectifs très ambitieux (accomplissement) ? Si les besoins sont compris et les attentes des salariés sont satisfaits, cela va sans doute, selon Maslow, booster la performance de l'organisation.

De sa part, MC GREGOR a élaboré une véritable théorie de management basée sur deux conceptions de l'homme au travail : la théorie $\mathrm{X}$ et la théorie $\mathrm{Y}$. Une approche pionnière dans l'explication de la catégorisation des salariés. En effet les individus ne sont identiques, cette hétérogénéité implique la segmentation des salariés en groupes homogènes en vue d'adapter les actions RH à leurs cibles.

Herzberg, estime que l'organisation scientifique du travail conduit à un sous-emploi des ressources humaines en méconnaissant ses potentialités. Il tente de repérer les éléments facteurs de satisfaction et d'insatisfaction au travail. Il en décèle 2 types :

Les facteurs d'ambiance - hygiène (bruit, chaleur, salaire, statut, relations humaines...) : Ils sont relatifs aux conditions de travail et doivent avoir un niveau de base. Si les conditions sont en dessous de ce niveau minimal, le travail s'en ressent, mais la productivité n'est pas influencée à la hausse si les conditions de travail sont supérieures au niveau de base. Ces conditions doivent être remplies pour ne pas générer d'insatisfaction.

Les facteurs valorisants - motivation (évolution de carrière, responsabilités, autonomie...) : ceux-ci correspondent aux besoins supérieurs de Maslow. Ils sont intrinsèques au travail et relèvent de l'épanouissement de l'individu. Une fois les facteurs d'hygiène assurés, les facteurs de motivation peuvent être remplis pour générer de la satisfaction.

Ces postulats théoriques confirment que l'amélioration de la qualité de vie au travail est sans conteste l'une des clés de la performance des organisations. Cette orientation capitale doit êtres inscrite dans un processus continu et adaptatif qui prend en compte les transformations du travail et de son organisation.

\section{I.2- Le lien entre le bien être au travail et performance économique}

Nous procédons à une revue de littérature portant sur les relations entre bien-être et performance dans l'objectif de dresser un état des connaissances.

Le chef d'entreprise est parfois tellement happé par le quotidien des contraintes liées à la vie de l'entreprise qu'il peut être amené à considérer que le bien-être de ses salariés est une préoccupation de second plan, voire un luxe qu'il ne peut se permettre. Pourtant, ce bien-être constitue le socle qui va permettre à l'entreprise de fonctionner de façon efficace et durable: engagement, réactivité, fluidité, esprit d'initiative. Au travers de cette étude, nous avons identifié principalement trois mécanismes qui relient le bien-être au travail à la performance économique:

\section{- L'amélioration de l'engagement des salariés}

Les coûts du désengagement ${ }^{3}$ sont souvent méconnus par l'entreprise alors que ceux-ci représentent un véritable manque à gagner. Le bon fonctionnement de l'entreprise ne repose pas seulement sur le respect de directives et de consignes, mais sur la capacité d'initiative de chacun face à des situations imprévues.

Plus on crée un environnement de «mieux-être », fondé sur le dialogue, l'écoute, le respect, l'honnêteté, sur un confort du lieu de travail, plus les salariés sont fidèles et défendent l'entreprise comme un bien commun. Ce mieux-être donne aussi aux salariés plus de possibilités de faire du bon travail.

\section{- L'instauration d'un fonctionnement propice à l'innovation :}

Intervenir sur le bien-être au travail, c'est également participer à l'amélioration de la créativité et de l'innovation. Le lien entre bien-être au travail et innovation n'est pas le plus manifeste et c'est en cela qu'il constitue aujourd'hui un vrai enjeu en terme de performance. En effet, lorsque certaines conditions sont réunies dans l'entreprise, il est possible d'accéder à de nouveaux potentiels d'innovation. Qui plus est, il s'agit d'une innovation souvent peu coûteuse. Certaines entreprises ont

${ }^{3}$ Le coût lié au remplacement d'un collaborateur (recrutement, intégration et formation de celui-ci), Le coût lié à la désorganisation, aux retards dans l'exécution du travail ...,Le coût lié à la négligence dans le travail (retards, erreurs, malfaçons ...),Le coût lié à la détérioration de l'image de l'entreprise 
parfaitement intégré ce levier dans leur stratégie managériale. Donc sans épanouissement, il n'y a pas d'efficacité. Il existe en effet un enchaînement logique «épanouissement, créativité, innovation ».

\section{- La diminution de l'absentéisme et du TURN OVER :}

Si la corrélation entre bien-être au travail et performance économique n'est pas toujours facile à chiffrer, un sentiment de mal-être se répercute en revanche de façon très tangible dans les résultats de l'entreprise : absentéisme, turn-over, accidents de travail, retards dans la production, défauts qualité etc... C'est un coût tout à fait conséquent que l'entreprise pourrait éviter.

En conclusion, rechercher les causes profondes à l'origine des phénomènes d'absentéisme et de turn-over améliore à la fois les conditions de travail des salariés et la performance de l'entreprise. Et cela représente un enjeu de taille sur le plan beaucoup plus large des dépenses liées à la protection sociale des salariés. S'intéresser aux raisons et mettre en place des actions correctives permettent une amélioration des conditions de travail et de la performance de l'entreprise.

En résumé, Il faut sortir du dilemme classique «bien-être des travailleurs » ou «performance économique de l'entreprise » car le bien-être est en réalité un facteur de performance.

\section{Section II : Bien-être au travail : une démarche complexe}

L'OMS définit le bien-être au travail comme « un état d'esprit caractérisé par une harmonie satisfaisante entre d'un côté les aptitudes, les besoins et les aspirations du travailleur, et de l'autre les contraintes et les possibilités du milieu de travail ».

\section{II.1. Le bien-être au travail : Paramètres et conditions de réussite}

Le bien être ne relève pas de la seule responsabilité de l'entreprise. Cette dernière se trouve dans un système et beaucoup de paramètres entrent en ligne de compte :

- La situation personnelle des collaborateurs : quand les salaries se sentent bien psychologiquement et physiquement sur leur lieu de travail ils sont plus épanouies et plus efficaces.

- Pandémies et crises économiques : Celles-ci entraîneraient une dégradation des conditions de travail nuit à la performance d'une entreprise et peut engendrer des incidents psychosociaux. Généralement, face aux crises les entreprises réduisent les heures de travail, rétrécissaient les actions réversibles en terme de (Salaires, formations, promotions) cela se répercute négativement sur la qualité de vie au travail (accidentes de travail, maladies, désengagement)

- Les relations employeurs / employés : les employés ont une bonne relation avec leur employeur ils réussissent à développer une relation de confiance qui permettrai de favorise le sentiment de bien être.

- L’âge : Les salariés ne sont pas exposés aux conditions de travail de façon uniforme selon l'âge. Les salariés vieillissants sont relativement moins exposés aux pénibilités et risques que leurs cadets ainsi à partir de 50 ans, diminue la proportion de salariés soumis à des efforts physiques, à des secousses ou vibrations, à des pénibilités visuelles ou à des températures extrêmes ${ }^{4}$. L'âge présente une relation linéaire avec le bien-être : plus nous vieillissons et plus nous nous sentons bien.

- Le genre : Le sexe amène peu de différences entre le bien-être des hommes et celui des femmes. Bien qu'il y ait une grande similarité du bien-être moyen entre les genres, l'articulation de ce dernier est cependant différente. Ainsi, les femmes vivraient une plus grande instabilité émotive, vivant ainsi un nombre d'affects négatifs et d'affects positifs plus important que les hommes. Ces derniers, vivraient des amplitudes émotives de moindre importance que chez les femmes, étant ainsi moins souvent très découragés ou très enthousiasmés. Mais malgré ces particularités, les niveaux de bien-être sont moyennement identiques.

\footnotetext{
${ }^{4}$ Ariane Pailhé, Âge et conditions de travail, Gérontologie et société 2004/4 (vol. 27 / n 111), pages 113 à 130.
} 


\section{II-2. Les conditions de la réussite de la démarche QVT}

Le succès d'une entreprise est intimement lié à la satisfaction professionnelle de ses employés. Un environnement professionnel où les employés se sentent valorisés, protégés, impliqués et écoutés, est propice au bien-être . Afin de permettre à ses employés de réaliser leur potentiel et d'évoluer, un employeur se doit de créer ce type d'environnement physique et immatériel.

Les conditions physiques qui permettent réussite d'une démarche du bien-être au travail :

- Il est primordial d'aménager un espace propice au confort d'une part, à la stimulation d'autre part. Concrètement, cela passe non seulement par l'aménagement des lieux - fauteuils de bureau ergonomiques, notamment - mais aussi par la décoration d'intérieur - plantes vertes, couleurs douces, meubles en bois brut... L'éclairage et la ventilation sont également des éléments importants pour se sentir bien au travail. Des locaux adaptés aux échanges, au travail en équipe, aux travaux spécifiques nécessitant plus de concentration individuelle,... et intégrant bien sûr les risques de sécurité...

- Une ergonomie du poste de travail qui préserve l'intégrité physique des salariés.

- des commodités pour simplifier la vie des salariées et rendre les lieux de travail plus attractifs

- Répondre aux besoins fondamentaux, ces besoins (outre des conditions matérielles de travail et de rétribution satisfaisantes évidemment) sont notamment environnement sain de travail.

\section{Des conditions immatérielles, constituent le moteur principal de la démarche QVT}

Ce qui favorise l'engagement des salariés, c'est le fait d'adhérer à des objectifs et d'être autonome dans la façon de les réaliser. Le fait de communiquer de façon ouverte et transparente en interne, concernant la vie et la situation de l'entreprise, donne à chacun du sens à son travail au quotidien, ce qui améliore d'autant son engagement et son efficacité.

Répondre aux besoins fondamentaux, Ces besoins (outre des conditions matérielles de travail et de rétribution satisfaisantes évidemment) sont notamment le Sens, la Reconnaissance, le Respect,

La question du sens est un des tous premiers piliers du Bien-être au travail : chacun au travail a besoin de se sentir utile car il cherche naturellement à comprendre pourquoi il fait ce qu'il fait.

La reconnaissance au travail s'appuie sur des valeurs de respect et d'appréciation des hommes et des femmes au travail, tant à propos de leur investissement personnel et de leurs résultats qu'à propos de leurs compétences ou de leur droit à la parole et à l'influence sur les décisions.

Pratiquer la reconnaissance au travail, c'est par exemple :

- Engager tous les salariés dans la recherche de l'innovation,

- Développer les groupes de résolution de problème,

- Consulter le personnel avant toute prise de décision

- Laisser prendre des initiatives à chaque collaborateur,

- Reconnaître les efforts de chacun, même si les résultats ne suivent pas toujours,

Le respect : s'ils le peuvent, les candidats choisiront des collègues et un patron avec lesquels ils prendront plaisir à travailler et desquels ils attendant du respect, que ce soit envers eux-mêmes, mais aussi envers leur idées et leurs atteintes, cette harmonie dans les relations professionnelles est la base de toute relation d'attachement, préalable à la fidélisation.

En créant un environnement propice à la prise en compte des besoins de chacun,

\footnotetext{
${ }^{5}$ Olivier Bachelard, Optimiser le bien-être au travail et la performance globale : enjeux et perspectives, Regards $2017 / 1$ $\left(\mathrm{N}^{\circ} 51\right)$, pages 169 à 179 .
} 
Donc finalement en agissant à la source, on gagne à la fois en bien-être, en fluidité, en créativité et on améliore le climat social ce qui permet au final un accroissement de la performance.

La démarche QVT ne révèle pas de la philanthropie. Le dirigeant qui prend ce genre de décision partage l'idée qu'un salarie qui va bien travaille mieux qu'un salarié qui va mal et il attend un certain nombre d'effets positifs pour l'entreprise, dont l'accroissement de l'efficacité au travail.

\section{Section III : Vérifications empiriques}

A travers cette enquête soumise à 250 cadres d'une organisation X, nous interrogerons sur la QVT afin d'évaluer ses retombées sur le rendement au travail. Ensuite un questionnaire adressé au staff de l'encadrement de la mémé entreprise permettra de croiser les résultats avec la performance. Un échantillonnage stratifié par domaine de compétence a été jugé nécessaire pour l'évaluation de la performance collaborateurs à des niveaux semblables.

Le choix de l'entreprise est justifié par son calcul systématique de la performance par des plateformes en ligne. Tandis que pour les collaborateurs le choix a été aléatoire.

\section{III-1-Généralités}

La ventilation des résultats se présente comme suit :

\section{Vous êtes :}

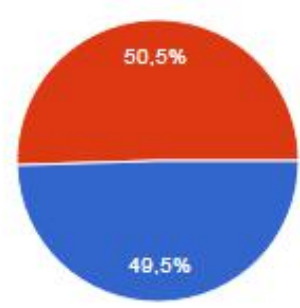

$$
\begin{array}{lll}
\text { Homme } & 50 & 49.5 \% \\
\text { Femme } & 51 & 50.5 \%
\end{array}
$$

Le nombre des hommes et des femmes qui ont répondu à ce questionnaire est presque égale à $50 \%$ des hommes et $51 \%$ des femmes.

Technique et mécanique

Logistique

Commercial

Finances

Ressources humaines

Achats

Transport

Informatiques et réseaux

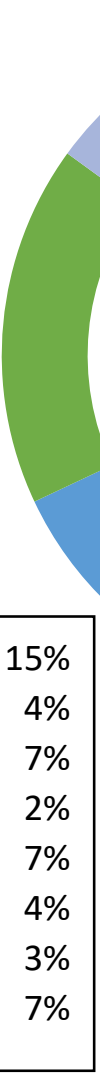

www.ijaers.com
Technique et mécanique

- Logistique

Commercial

Finances

Ressources humaines

Achats

- Transport

- Informatiues et réseaux

medecine de travail

Relations avec la clientèle

- Production 1

Production 2

Production 3

\begin{tabular}{|lr|}
\hline Médecine de travail & $1 \%$ \\
Relations avec la clientèle & $6 \%$ \\
Production 1 & $12 \%$ \\
Production 2 & $17 \%$ \\
Production 3 & $15 \%$ \\
\hline
\end{tabular}


Pour évaluer La perception de la QVT nous nous intéressons principalement aux thèmes suivants :

Conditions immatérielles : la capacité d'expression et d'action dans le cadre de leur travail,

le climat social, l'ambiance au travail, les relations positives avec les autres, le leadership, acceptation de soi, communication interne, autonomie, la motivation aux tâches.

Conditions physiques : conditions général d'emploi et de travail

Le questionnaire interrogeant des salariés sur les éléments qui influent sur leur perception de la QVT sont très révélatrices : les relations humaines (avec leurs collaborateurs et avec le management) sont les premiers éléments cités. Viennent ensuite les éléments liés à la reconnaissance et au développement/progression dans le travail ensuite la conciliation entre la vie personnelle et la vie professionnelle.

\section{III-2- Les conditions physiques de travail et la qualité de vie au travail}

\section{Nous avons retenu les thèmes suivants :}

- Conditions générales de travail : contrats, salaires, hygiène et la santé sécurité au travail

- L'intérêt au travail (contenu des tâches)

- L'ambiance du travail

- Les relations hiérarchiques

Q 1: Actuellement, au sein de l'entreprise / de l'administration dans laquelle vous travaillez, diriez-vous que le climat social est :

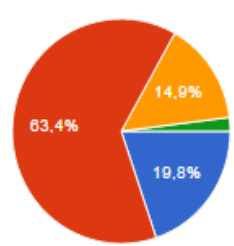

$$
\begin{array}{rrr}
\text { Très bon } & \mathbf{2 0} & 19.8 \% \\
\text { Assez bon } & \mathbf{6 4} & 63.4 \% \\
\text { Assez mauvais } & \mathbf{1 5} & 14.9 \% \\
\text { Très mauvais } & \mathbf{2} & 2 \%
\end{array}
$$

On remarque que la majorité des interviewés trouvent que le climat social au sein de leur domaine de travail est assez bon $(63,4 \%)$. Cela peut être expliqué par l'impact des facteurs internes et externes sur le degré de satisfaction qui règne dans l'entreprise.

Le climat social peut être influencé par :

Des facteurs internes : stress, satisfaction des salariés....

Des facteurs externes : crise économique, augmentation du chômage, baisse de consommation....

\section{Q 2 : Aujourd'hui, si vous deviez noter de 1 à 10 votre bien-être au travail quelle note donneriez-vous ?}

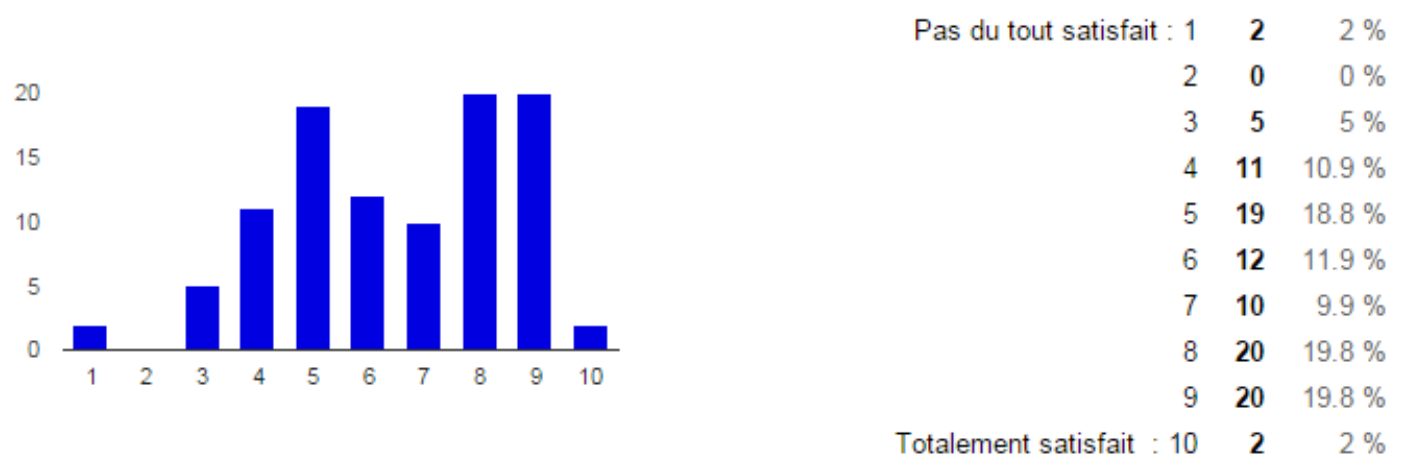

D'après notre analyse de ce graphique qui représente le degré de la sensation du bien-être au travail des employés, on constate que : 
$2 \%$ sont ne se sentent pas satisfaits : ceux-ci peut être expliqué par l'absence des facteurs relatifs aux conditions fondamentales du travail : sécurité de poste, salaire, déclarations sociales...etc.

Ces derniers sont considérés comme des conditions pathogènes qui se situent à la frontière entre le travail et la santé :

Sécurité de travail, hygiène du travail, aspects psychosociaux...

$18,8 \%$ : se sentent moyennement satisfaits et $52 \%$ très satisfaits : ceux-ci montre que les conditions vécus par les employés sont favorables ou au moi répondent à leurs attentes.

\subsection{La motivation au sein de l'entreprise}

\section{Q 3 : Actuellement, vous sentez-vous dans votre travail ?}
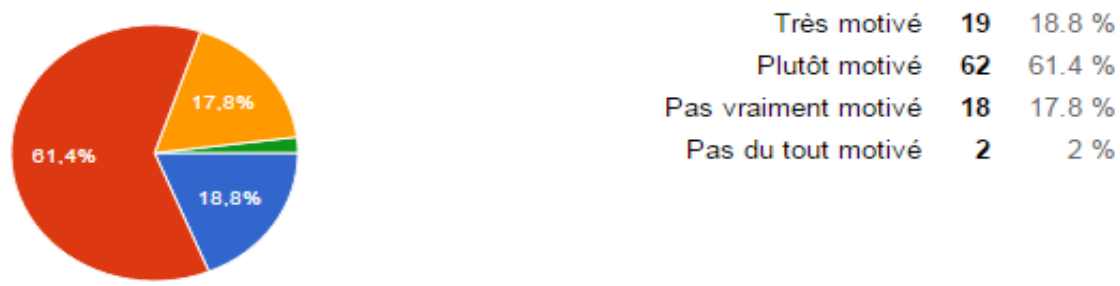

On constate que la plupart des salariés se sentent motivés $(80,2 \%)$ : C'est-à-dire que les avantages matériels, conditions sociales ... offerts par l'entreprise répondant aux attentes des salariés.

Un restreint pourcentage $(19,8 \%)$ des employés se sentent démotivés par rapports aux conditions fondamentales : Flexibilité du travail, aménagement des lieux de travail, les conditions de l'hygiène au travail,

\section{$\underline{\text { 3-3- Mesure de la satisfaction au travail }}$}

Vos conditions matérielles de travail [Q 4 : Etes-vous très, plutôt, plutôt pas ou pas du tout satisfait de :]

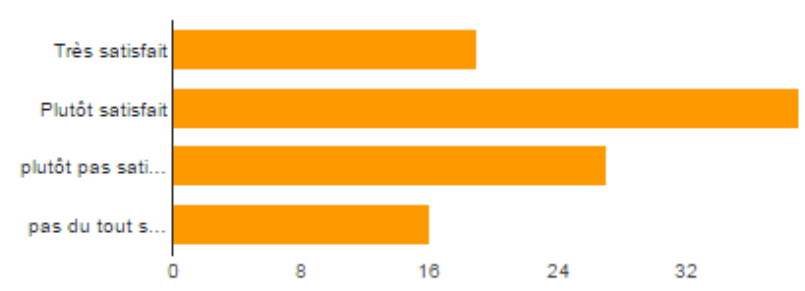

$\begin{array}{rll}\text { Très satisfait } & \mathbf{1 9} & 18.8 \% \\ \text { Plutôt satisfait } & \mathbf{3 9} & 38.6 \% \\ \text { plutôt pas satisfait } & \mathbf{2 7} & 26.7 \% \\ \text { pas du tout satisfait } & \mathbf{1 6} & 15.8 \%\end{array}$

Ensemble des salariés [Q 4 : Etes-vous très, plutôt, plutôt pas ou pas du tout satisfait de :]

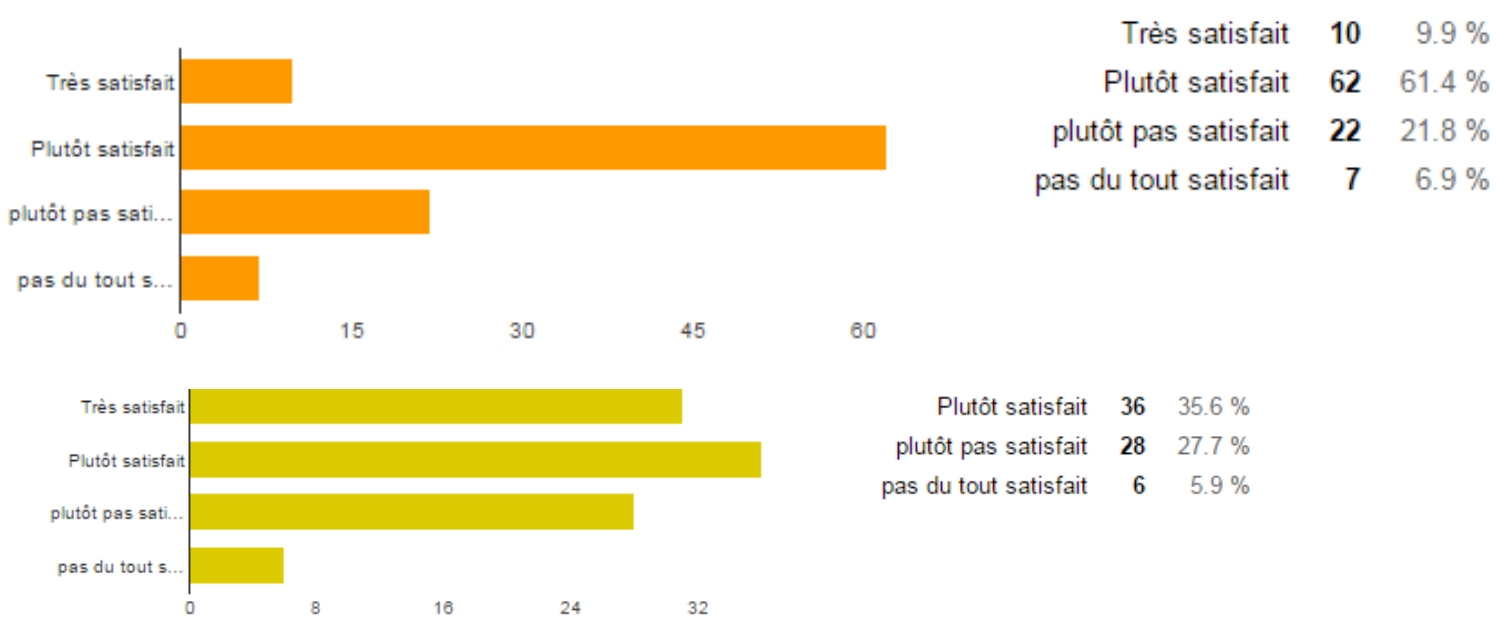

Seulement $10 \%$ des enquêtés sont hyper-satisfait, $84 \%$ avoisinent le point d'indifférence et presque le $1 / 3$ insatisfaits peut être expliqué par : l'absence ou l'existence des conditions de favorables au travail. Ils se plainent essentiellement de la charge du travail, des horaires qui ne les conviennent pas surtout les femmes (68\%). 
D'une autre part, la performance ne dépasse guère $48 \%$ mesuré par le degré d'atteinte des objectifs organisationnels (durant le mois n-1 des salariés en questions).

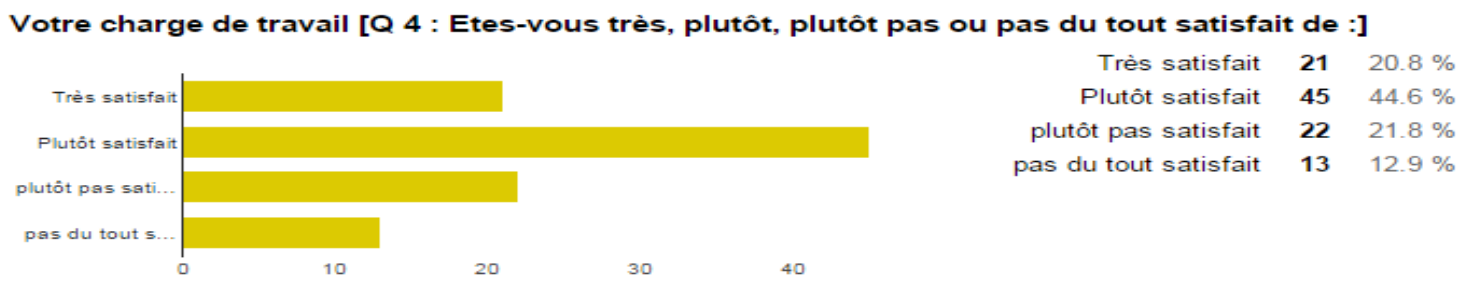

\section{3-4. La qualité des relations entretenues avec les autres}

La majorité des employés sont plutôt satisfait des la relations avec les collègues du travail et uniquement $4 \%$ qui sont pas du tout satisfaits ; ceux-ci montre que les conflits et les litiges entre les salariés ne sont pas fortement présents au niveau des entreprises.

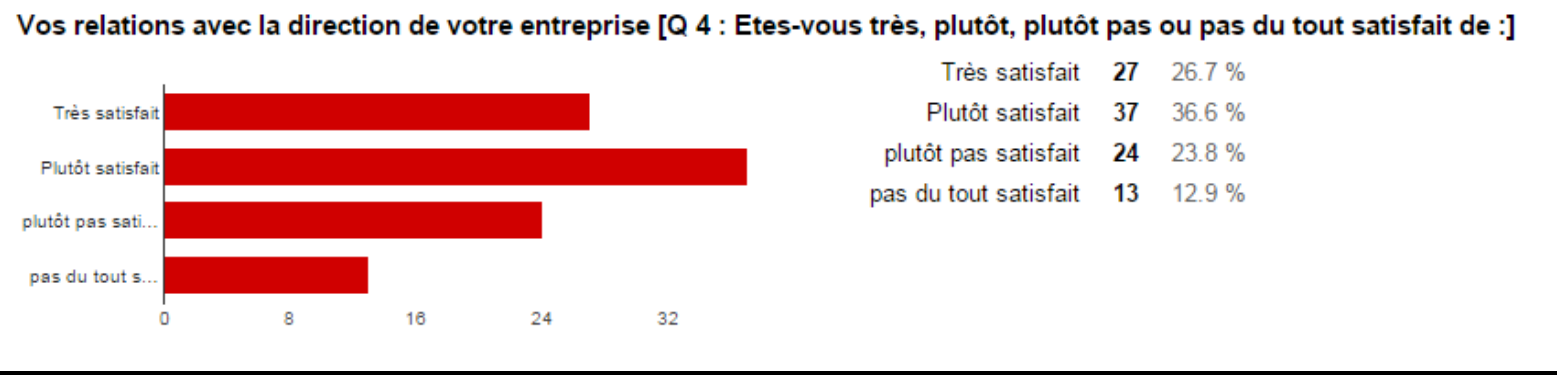

Généralement les interviewés ne se plainent pas des relations entretenues avec les autres, les conflits hiérarchiques sont maintenus à des niveaux bas et des relations chaleureuses sont entretenues horizontalement et verticalement. La clé de réussite est justifiée par la clarté et la transparence des procédures de travail (généralement formalisées), Des actions de team building, des ateliers Yoga de rire,...etc en vue d'entretenir des liens favorables au travail.
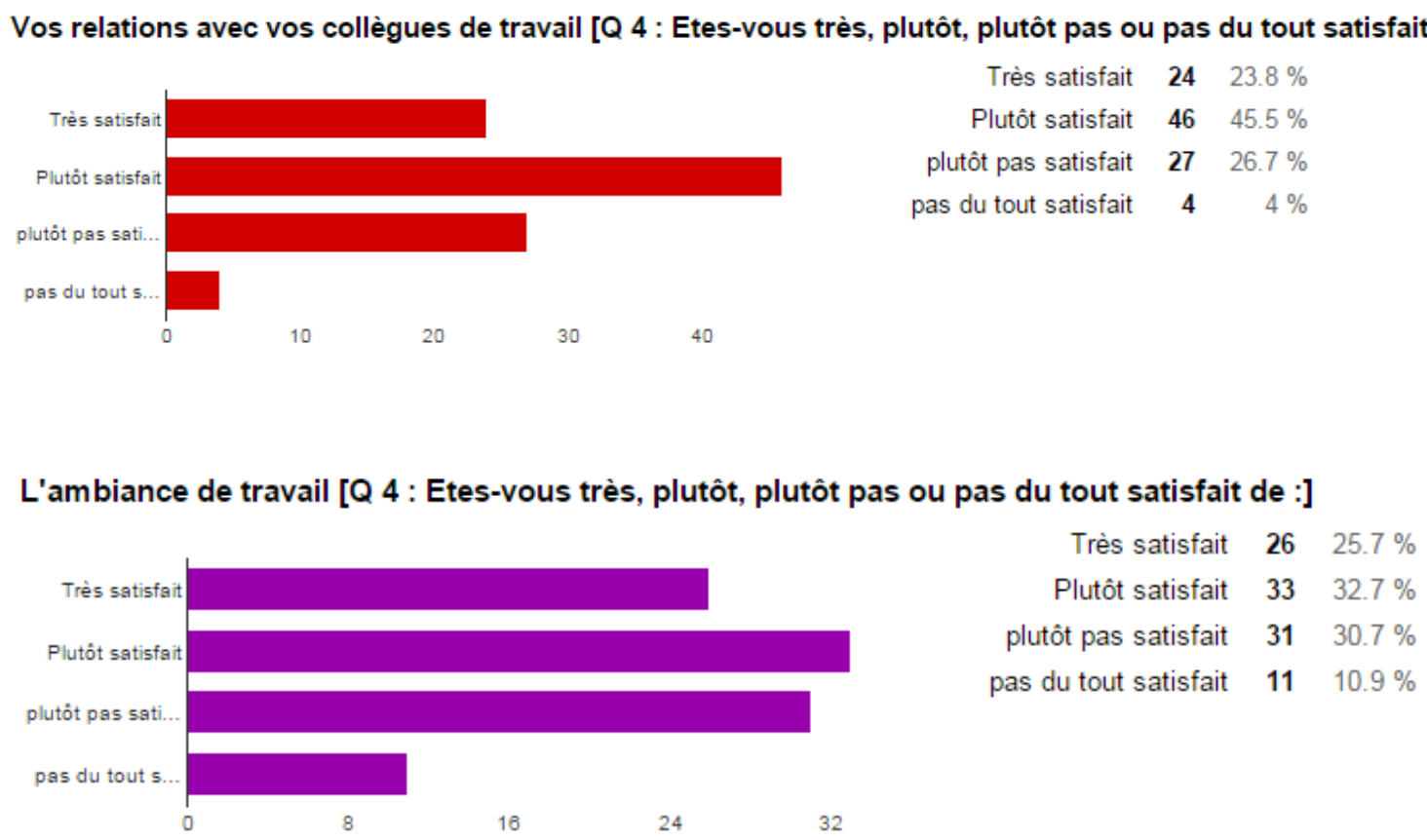
(généralement le personnel tachnique) critiquents plutôt les conditions de travail : Aération des ateliers, les condtiions liées à la sécurité et à l'hygiéne au travail.

Nous avons interogés dans ce sens l'encadrement qui a avoué de la détérioration de certains équipements ainsi que de la nécessité de revoir les conditions d'exercice dans certains ateliers ainsi les équipements de protection des callaboreteurstechnique. De sa part, le comité de sécrurité et d'hégiéne avait soulevé ces remarques dans ces rapports précédents. Par ailleurs, il était difficile d'établir le lien avec le rendrement des salariés, étant donné que d'autres facteurs techniques interviennent: Equipeements, matiéres premiéres..etc. ce qui est intéressant, cest que nous avons soulevé un écart remarquable entre le rendement des salariés pendant la prise de nuit. Cela explique evidement le lien étroit entre les plages horraires de travail et la performance des salariés.

\section{III-3-Le facteur pshycologique : est il explicatif de la performance ?}

De nombreux facteurs tels que l'hyperconnexion des salariés, ou encore l'intensification du travail perturbent cet équilibre fragile. Un déséquilibre pouvant entraîner du stress, voire un burn-out.

- L'équilibre entre vie privée et professionnelle

- La transparence des pratiques RH

- Fluidité de la communication

- Accès à la formation

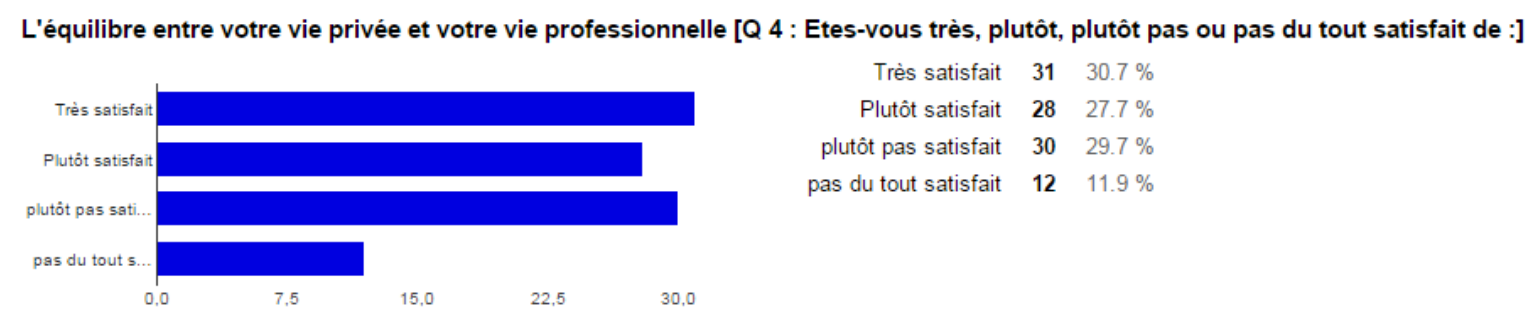

Q 5 : D'une manière générale, vous sentez-vous bien ou mal informé(e) sur ce qui se passe au sein de votre entreprise ?

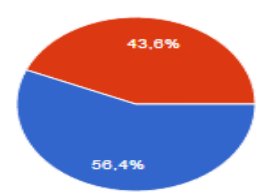

Bien informé $57 \quad 56.4 \%$ Mal informé $\mathbf{4 4} \quad 43.6 \%$

Commentaire ; Concernant la circulation de l'information dans l'entreprise :

La moitié des employés de notre échantillon (100 personnes) ont répondu qu'ils sont bien informés : ceux-ci montre que : l'information circule bien dans l'entreprise, néanmoins il faut souligner que l'entreprise doit communiquer la bonne information au bon moment et à la bonne personne ;

Q 6: Au cours de l'année écoulée avez-vous bénéficié d'une ou plusieurs formation(s) professionnelles dans le cadre de votre travail ?

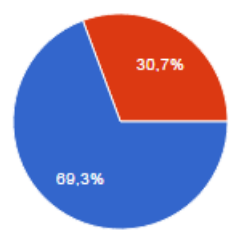

Oui $70 \quad 69.3 \%$

Non $31 \quad 30.7 \%$

Commentaire ; Concernant les formations professionnelles 70 personnes ont répondu positivement sur le fait qu'ils bénéficient vraiment de ces dernières au sein de leur domaine de travail ; c'est une réponse favorable, au sens où l'employé va pouvoir acquérir des savoirs faire nécessaires à l'exercice de son métier. 
Q 7 : Les formation(s) dont vous avez bénéficiée(s) :
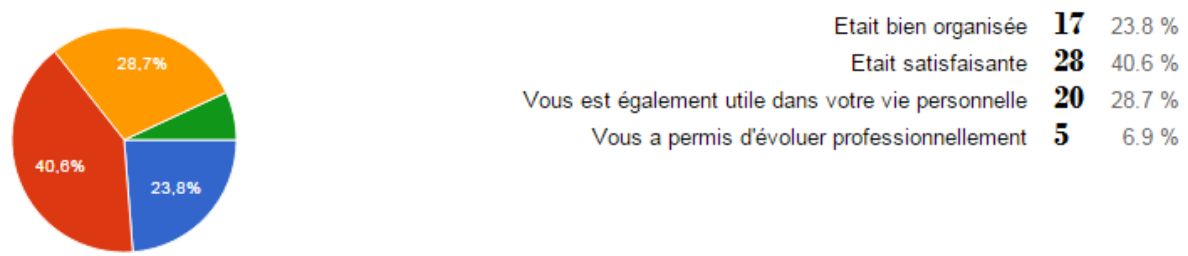

Commentaire ; Presque $41 \%$ employés ont répondu que ces formations professionnelles ont été satisfaisantes, on peut dire qu'ils ont pu apprendre les connaissances et les compétences nécessaire à l'exécution de leur activité courante.

\subsection{La communication interne au sein de l'entreprise :}

\section{Q 8 : Globalement, quelle note donneriez-vous à la communication interne de votre entreprise dans son ensemble ?}

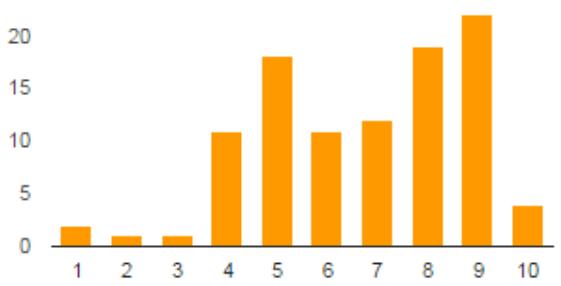

$\begin{array}{rrr}\text { pas bonne du tout : } 1 & 2 & 2 \% \\ 2 & 1 & 1 \% \\ 3 & 1 & 1 \% \\ 4 & 11 & 10.9 \% \\ 5 & 18 & 17.8 \% \\ 6 & 11 & 10.9 \% \\ 7 & 12 & 11.9 \% \\ 8 & 19 & 18.8 \% \\ 9 & 22 & 21.8 \% \\ \text { très bonne }: 10 & 4 & 4 \%\end{array}$

Commentaire ; Le 9ème degré a été le plus répondu pour la question de la communication interne dans l'entreprise (22 personnes parmi 101) : Ceux-ci reflètent un déficit apparent qui touche la communication interne, ce déficit va se répercuter sur le fonctionnement de l'entreprise au quotidien, il est nécessaire de développer les outils de communication interne tel que :

Le journal de l'entreprise, l'affichage interne, le flash d'information, l'organigramme de l'entreprise, les notes de service....

\section{DISCUSSIONS ET CONCLUSION}

Nous avons adopté une méthodologie précise pour prouver le lien entre la qualité de vie au travail et la performance. Nous nous sommes d'abord référés aux cadres-salariés. La question du bien-être au travail et de ce qui s'y rapporte, pour en comprendre l'articulation et dessiner un cadre conceptuel. Une fois ces recherches effectuées, nous nous sommes concentrés sur le terrain d'actions, qu'est celui de l'entreprise X. Après en avoir analysé le fonctionnement passé, présent mais aussi futur, nous nous sommes concentrés sur les ressentis des membres de la direction. Les entretiens que nous avons menés avec la plupart des membres se sont avérés être une immense richesse. Il est vrai que nous aurions peut-être pu étendre ces entretiens qualitatifs semi-directifs à tous les membres de la direction et aux différents managers, mais notre immersion au sein du l'entreprise X était limitée.

Etudier les leviers de la performance pousse à s'interroger sur les causes de la non-performance ; classiquement on retient les 4 causes suivantes : le taux d'absentéisme, le taux de Turnover, les Non-qualités et l'inefficacité au travail. Au delà des phénomènes de corrélation et d'amplification liant entre-eux ces 4 paramètres, il est très fréquent de découvrir une origine commune : le désengagement et la perte de motivation chez les personnes.

L'amélioration de la qualité de vie au travail pemert de pallier à ces facteurs. Ainsi les actions qui permettent de préserver la santé des salariés et d'éloigner les risques (risques psychosociaux notamment). Elle favorise leur engagement individuel et collectif. Assiduité, motivation, productivité, créativité, émulation, fierté d'appartenance... Autant de leviers indispensables à l'épanouissement du salarié, en tant que travailleur et individu.

Dans une dynamique gagnant-gagnant, et parce qu'elle permet une meilleure efficacité sur le long terme, la QVT profitera aussi à l'employeur. Des conditions de travail peu favorables auront, à l'inverse, des répercussions directes sur l'entreprise : 
fort taux d'absentéisme, retards dans la production, désorganisation du travail, recours à la sous-traitance ou à des contrats temporaires, hausse des cotisations versées à la Sécurité sociale, turnover...

Les résultats obtenus montrent que la qualité de vie au travail impacte la performance au travail. En revanche, le facteur physique est plus significatif que les facteurs phycologiques

Parce qu'elle met en relation qualité de vie au travail et performance, la QVT oblige à repenser ce que l'on entend par "performance(s)". Sur quels critères une entreprise peut-elle évaluer son efficacité ? La mesure de la performance se réduitelle à des chiffres et à une suite d'indicateurs financiers ?

Tout ces facteurs peuvent influencer sur la situation de l'employé au sein de l'entreprise, c'est pour cela que la plupart des réponses recueillies par cette enquête ont démontré un faible degré de satisfaction et de motivation dans l'entreprise quant au facteur physique, en conséquence la performance de l'entreprise sera très touché par le mal être de l'employé.

Donc, quand le salarié se sent entouré par les bonnes conditions de travail, il va fournir plus d'effort dans son milieu de travail.

En guise de conclusion on peut conclure que le lien entre le bien-être au travail et la performance économique est vérifié. L'impact du facteur physique est plus significatif.

L'amélioration des conditions de travail se décline de plus en plus dans la recherche du bien-être et permet même d'améliorer la performance des entreprises ; remettre du bien-être au travail s'inscrit alors dans une perspective de respect de l'individu et du l'efficacité économique.

\section{BIBLIOGRAPHIE}

[1] CERCLE D’ETHIQUE DES AFFAIRES. Le bien-être au travail. Paris : Ethique édition, 2010, 104 p.

[2] COTTRAUX Jean. Psychologie positive et bien-être au travail. Issy les Moulineaux : Elsevier Masson, 2012, 202 p.

[3] DUMAS Marc. Vie personnelle et vie professionnelle : vers un nouvel équilibre dans l'entreprise ? Cormelles-le-Royal : Editions EMS, 2008, 173 p.

[4] GRESY Jean-Edouard, PEREZ NÜCKEL Ricardo, EMONT Philippe. Gérer les risques psychosociaux : performance et bien-être au travail. Issy les Moulineaux : ESF, 2012, 223 p.

[5] KENNEDY Carol. Toutes les théories du management : les idées essentielles des auteurs les plus souvent cités. Paris : Maxima, 2008, $444 \mathrm{p}$.

[6] LETHIELLEUX Laëtitia. L'essentiel de la GRH. Paris : Gualino-Lextenso éd, 2011, 116 p.

[7] PORTELLA Angela. Repenser le bien-être au travail. Levallois-Perret : Vocatis, 2010, 149 p.

[8] ROJOT Jacques, ROUSSEL Patrice, VANDENBERGHE Christian et al. Comportement organisationnel Volume 3. Bruxelles : De Boeck, 2009, 396 p.

[9] STEILER Dominique, SADOWSKY John, ROCHE Loïck. Eloge du bien-être au travail. Grenoble : Presse Universitaire de Grenoble, 2010, 104 p.

[10] THEVENET Marc. Le plaisir de travailler : favoriser l'implication des personnes. Paris : Editions d'organisation, 2000,269 p.

[11] VITARI Claudio, ASHTA Arvind, BLOEMMEN Marjolijn et al. Slow Management : entreprendre la transition. Paris : Pearson, 2013, $235 \mathrm{p}$.

[12] ANGEL Pierre Angel « Développer le bien-être au travail : Stress, épuisement ; harcèlement.. ». Une réponse innovante : les programmes d'aide aux salariés Edition DUNOD P. $92-98$

[13] MOYAT-AYCOBERRY Guide des risques psychosociaux en entreprise $3^{e}$ édition, Dispositifs juridiques - Leviers d'action - Fiches pratiques, Editeur: GERESO Nombre de pages : 139

[14] ENRIQUEZ, E. (1997). "Les enjeux éthiques dans les organisations modernes" In Les enjeux du pouvoir et du désir dans l'entreprise, Paris, Desclée de Brouwer, 115-137

[15] DEVLEESCHOUWER, A. (2010). Impact du bien-être et de la motivation au travail sur la performance in-rôle et les comportements de citoyenneté organisationnelle chez les vendeurs en télécommunication. Mémoire de maitrise, Louvain-la-Neuve, Université catholique de Louvain

[16] Ariane Pailhé, Âge et conditions de travail, Gérontologie et société 2004/4 (vol. 27 / n 111), pages 113 à 130. 
[17] Olivier Bachelard, Optimiser le bien-être au travail et la performance globale : enjeux et perspectives, Regards 2017/1 ( $N^{\circ} 51$ ), pages 169 à 179.

\section{Articles scientifiques}

[18] ABADIA G., Delépine A., Hache P. et al. 32ème congrès national de médecine et de santé au travail. Références en santé au travail, décembre 2012, n 132 , p. 47 - 57.

[19] LAPERRIERE Eve, MESSING Karen, BOURBONNAIS Renée. « Pour être serveuse, tu dois avoir toute ta tête » : efforts et reconnaissance dans le service de table au Québec. Travailler, 2010, n 23, p. 27 - 56.

[20] GROSJEAN V., FORMET N., ALTHAUS V. et al. Recherche et développement conceptuels autour du bien-être au travail : entre mesure et action. Références en santé au travail, septembre 2014, n 139 , p. 29 - 39.

[21] MORIN Estelle M, GAGNE Charles. Donner un sens au travail, promouvoir le bien-être psychologique. IRSST, 20 p.

[22] RICHER Martin. «Bien-être et efficacité » : pour une politique de qualité de vie au travail. Terra Nova, 18 mars 2013,22 p.

[23] VAILLANT Nicolas, WOLFF François-Charles. Stress, anxiété et dépression au travail : existe-t-il des différences entre entreprises ? Revue française d'économie, 2010, n 4 volume XXV, p. 39 - 74.

[24] Revue-des-sciences-de-gestion-2010-1-page-117

[25] Rapport du chambre des salariés de Luxemburg, Une analyse des données du Quality of work Index Luxembourg Newsletter $\mathrm{N}^{\circ}$ 2/2015, 16 mars 2015 